\title{
Evaluating Place-based Accessibility using Space-time Utility Perspective
}

\author{
William H. K. Lam ${ }^{1}$, Bi Yu Chen ${ }^{1,2}$, Eric J. Miller ${ }^{3}$ \\ ${ }^{1}$ Department of Civil and Environmental Engineering, the Hong Kong Polytechnic University \\ Hong Kong, China \\ william.lam@polyu.edu.hk \\ ${ }^{2}$ State Key Laboratory of Information Engineering in Surveying, Mapping and Remote Sensing, Wuhan University \\ Wuhan, China \\ chen.biyu@whu.edu.cn \\ ${ }^{3}$ Department of Civil Engineering, University of Toronto \\ Toronto, Canada \\ miller@ecf.utoronto.ca
}

\begin{abstract}
The conventional static place-based accessibility measures are commonly used in practice for evaluation of transport network performance. However, this conventional approach have often been criticized for ignoring the dynamics in human space-time behaviours and urban environments. In this study, dynamic place-based accessibility measures are proposed to extend the conventional place-based measures by combining the merits of the place-based and space-time utility-based approaches. In this new approach, the accessibility of a place within the study area can be evaluated by the space-time utility approach in terms of activity duration, travel time and facility attractiveness. The individual choice behaviours amongst multiple alternatives are explicitly considered in the placebased accessibility evaluation. To demonstrate the implementation and applicability of the proposed place-based accessibility measures, a comprehensive case study is carried out in Wuhan which is one of the fast-growing mega cities in China.
\end{abstract}

Keywords: Accessibility, Space-Time Utility, Time Geography, Transport Network Performance.

\section{Introduction}

Accessibility is an important concept for urban planning, transport geography, and other related fields. It is defined as the ease with which urban services can be reached from a particular place or location (or by individuals at that location) using a particular transport system [1-2]. Poor accessibility to urban services (e.g., jobs, recreational services, healthcare facilities, etc.) can negatively impact the citizens' quality of life, and even lead to social exclusions in extreme cases. Accessibility to urban services has been intensively studied in the literature not only for various strategic planning purposes (e.g., transport network performance evaluation) [3-5], but also for being an explanatory factor in many geographic phenomena analysis (e.g., social equity and justice) [6-8].

The evaluation of accessibility to urban services depends on accessibility measures. In the literature, various measures have been developed and can be roughly classified into two categories, including place-based and individual-based measures [2,9]. Conventional place-based measures conceptualized accessibility largely in terms of the proximity to urban services from an individual's residential location. The widely used place-based measures include the travel distance to the nearest service location, the cumulative number of services within a specified cut-off distance, gravity-type measures in which the attractiveness of services decreases with the distance from the origin $[10,11]$. These conventional place-based measures required a few of aggregated data and can be easily applied for large-scale study areas. However, they are static spatial terms and often criticized for ignorance of dynamics in human space-time behaviours and urban environments [9, $11,12]$.

In the past decade, it has become easier to move beyond the place-based accessibility measures due to the availability of individual-level activity diary data $[2,9,12]$. In the literature, several individual-based (or space-time) measures have been proposed. Most of them are built on the time geographic framework [13] for capturing complexities of individual activity-travel behaviours under various space-time constraints. The number of reachable urban services and the 
cumulative activity durations at reachable urban services are two well-known individual-based measures [10]. Theoretically, a better approach is the space-time utility measures that can directly evaluate individual utilities of activity participation in terms of activity duration, travel cost and the facility attractiveness [14]. The individual choice behaviours amongst multiple alternatives, such as logsum, can be explicitly considered. Several advantages of using space-time utility approach, particularly log-sum, for the individual accessibility evaluation are discussed and summarized in [15]. All these individual-based measures are well suited to evaluate accessibility to urban services for people in different social groups and geographical regions [1]. However, they require detailed individual-level activity diary data, which can be very expensive and difficult to acquire large number of samples. As a result, previous studies used individual-based measures are restricted to a relatively small area using a few samples.

In recent years, increasing attention has been given to improve the conventional place-based measures with individualbased perspectives. Páez et al. [16] improved conventional cumulative-opportunity measure by using model estimated average travelled distance of people in different social groups and geographical regions. Delafontaine et al. [17] proposed enhanced place-based measures by considering the facility's opening hours and activity durations at facilities. Widener et al. [18] and Fransen et al. [19] extended conventional place-based measures by using interaction potential metrics with consideration of inter-zonal commuting patterns.

In view of this trend, this study aims to develop a dynamic operational accessibility evaluation framework with integration of the advantages of both the place-based and space-time utility-based approaches. In this study, the accessibility of a location is evaluated by the space-time utility approach in terms of activity duration, travel time and facility attractiveness. The individual choice behaviours amongst multiple alternatives are explicitly considered in the place-based accessibility evaluation. Therefore, the proposed place-based utility measures can enhance the ability to realistically evaluate place-based accessibility by using the robust space-time utility approach. To demonstrate the operationalization and applicability of the proposed place-based utility measures, a comprehensive case study is conducted in Wuhan which is one of the mega cities in China. Two types of spatiotemporal big data, including check-in data and taxi tracking data, are collected for case study. Check-in data of a popular social media site are extracted to estimate the timedependent attractiveness function of facilities. Large-scale taxi tracking data are collected to estimate time-varying travel times in Wuhan road network. The results of the case study indicate that the proposed place-based utility measures can full capture the temporal variations of place-based accessibility due to both time-varying travel times and attractiveness of facilities. The results of the case study also demonstrate the potential usefulness of merging spatiotemporal big data for large-scale accessibility studies.

The remainder of this paper is structured as follows. In the next section, the conventional place-based accessibility measures are briefly introduced to provide the background of the problem. The proposed place-based utility measures are then presented in Section 3. The case study in Wuhan is reported in Section 4. Finally, conclusions are given in Section 5 together with recommendation for further studies.

\section{Conventional Place-Based Accessibility Measures}

Accessibility has traditionally been evaluated by place-based accessibility measures. For instance, the widely used cumulative-opportunity measure [20], denoted by $C U M$, is represented by the number of facilities within the standard travel time polygon (TTP). As illustrated in Figure 1, TTP delimits all possible geographical locations, where the travel time from the individual's residential location $r$ is less than given travel time budget $b$. It can be expressed as

$$
T T P=\left\{x \mid t_{r x} \leq b\right\}
$$

where $t_{r x}$ is the travel time from an individual's residential location $r$ to location $x$. Let $f_{i}{ }^{j}$ be a facility for conducting activity $j$. The set of facilities within the TTP forms the choice set $F O S=\left\{\forall f_{i}^{j} \in T T P\right\}$. Then, the $C U M$ measure for activity $j$ can be expressed as

$$
\operatorname{CUM}(j)=\sum_{\forall f_{i}^{j} \in F O S} 1
$$


Another commonly used place-based accessibility measure is the following gravity-type measure (denoted by $G R A V$ ):

$$
G R A V(j)=\sum_{\forall f_{i}^{j} \in F O S}\left(a_{f_{i}^{j}}\right)^{\alpha^{j}} \exp \left(-\lambda^{j} t_{r f_{i}^{j}}\right)
$$

where $a_{f_{i}{ }^{j}}$ is attractiveness of facility $f_{i}{ }^{j}, t_{r f_{i}{ }^{j}}$ is the travel time from residential location $r$ to facility $f_{i}{ }^{j}$. Attractiveness $a_{f_{i}^{j}}$ is measured by a multitude of attributes related to the facility, such as size, services offered, waiting times, etc. Sensitive parameters, $\alpha^{j}$ and $\lambda^{j}$, are related to attractiveness and the friction of distance to the facility for conducting activity $j$, respectively. Comparing with $C U M(j)$, this $G R A V(j)$ measure incorporates two additional components: the facility attractiveness and the friction of distance to the facility, expressed by the distance-decay function. A negative exponential decay function is discussed here, because this is one of the most widely used distance-decay functions in the accessibility literature. For the use of other functions, interested readers may refer to [10].

Although these place-based measures are valuable for evaluating accessibility of large-scale study areas, they are often criticized for their ignorance of dynamics in human space-time behaviours and urban environments. In next section, these conventional place-based measures are improved by incorporating the time dimension, in terms of activity durations and accessibility temporal variation at different times of the day.

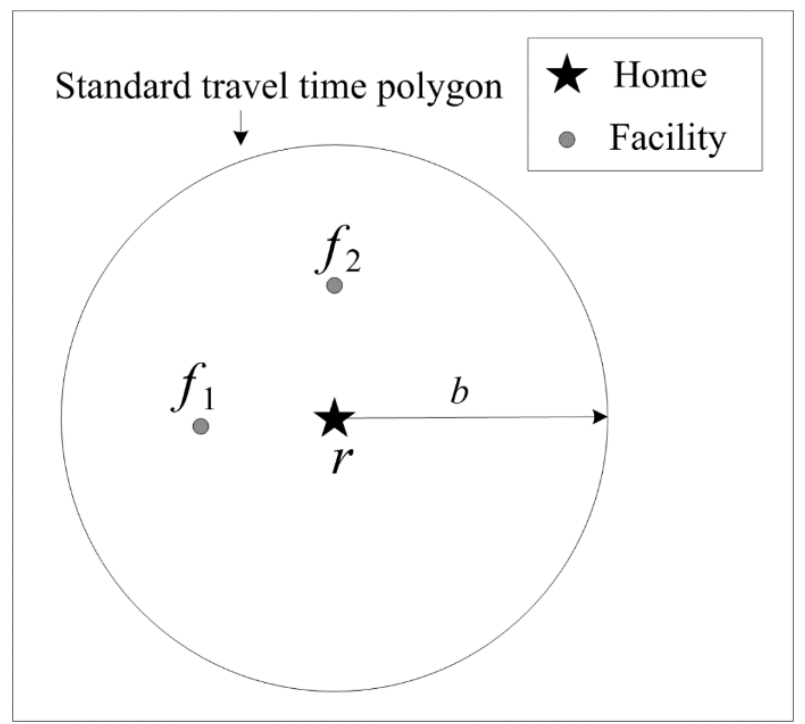

Fig. 1: Standard travel time polygon concept.

\section{Proposed Place-Based Space-Time Utility Measures}

Following the time geographic approach, given an individual living at residential location $r$, with a time budget from $t_{r}$ to $t_{s}$, all possible space-time locations for the individual's activity participations can be expressed as the forward spacetime cone, $F C(t)$, (Miller, 2005):

$$
F C(t)=\left\{x \mid t \geq t_{r}+t_{r x}, t_{r x} \leq t_{s}-t_{r}-c_{\min }, t_{r} \leq t \leq t_{s}\right\}
$$

where $t_{r x}$ is the travel time from residential location $r$ to location $x$; and $c_{\min }$ is the minimum activity duration required for activity participations. Figure 2 illustrates this space-time cone concept in three-dimensional (3D) space. The height of space-time cone at location $x$ indicates the maximum duration $c_{x}$ for activity participations at the location, which can be written as: 


$$
c_{x}=t_{s}-t_{r}-t_{r x}
$$

To successfully participate in activity at the location, the maximum duration should satisfy the $c_{x} \geq c_{\min }$ constraint. The projection of space-time cone onto the two-dimensional (2D) geographical space is the potential path area, which is essentially identical to the standard travel time polygon:

$$
T T P=\left\{x \mid t_{r x} \leq t_{s}-t_{r}-c_{\min }=b\right\}
$$

where travel time budget $b=t_{s}-t_{r}-c_{\min }$. Therefore, $F C(t)$ shown in Figure 2 extends TTP shown in Figure 1 by incorporating the time dimension in terms of activity durations; and FOS defined by $F C$ is identical to that defined by the above TTP .

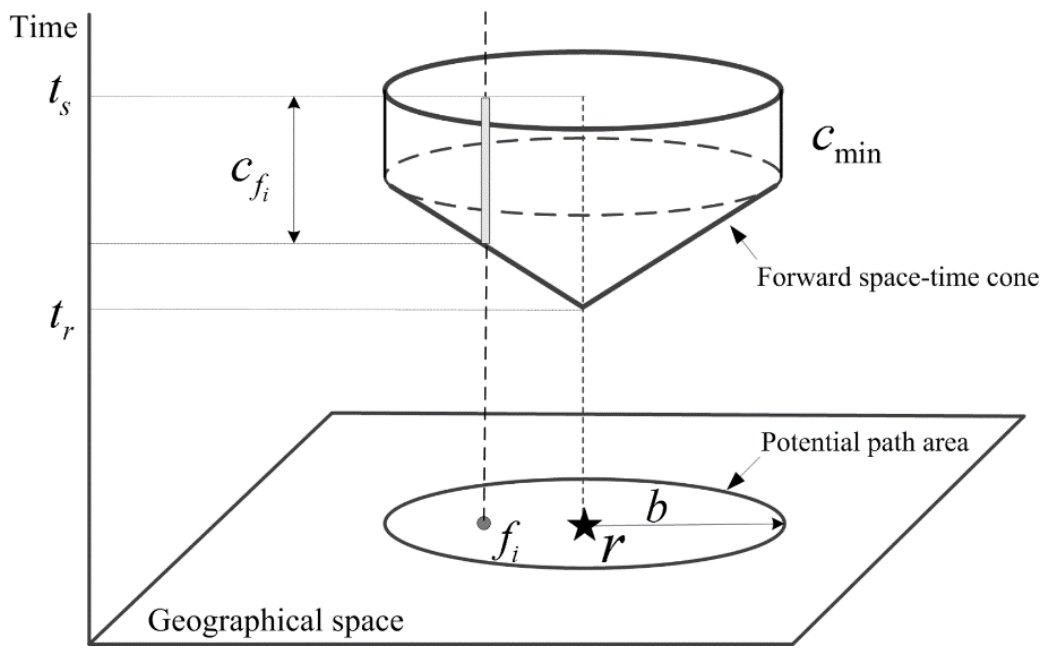

Fig. 2: Forward space-time cone concept.

Following the space-time utility approach [14], given a facility $f_{i}^{j} \in F O S$, the direct benefits (or utility) of activity participations at the facility can be measured in terms of attractiveness of the facility $a_{f_{i}}$, travel time $t_{r f_{i}^{j}}$ and activity duration $c_{f_{i}{ }^{j}}$ as:

$$
U_{f_{i}^{j}}(j)=\left(a_{f_{i}^{j}}\right)^{\alpha^{j}}\left(c_{f_{i}^{j}}\right)^{\beta^{j}} \exp \left(-\lambda^{j} t_{r f_{i}^{j}}\right)
$$

where $\alpha^{j}, \beta^{j}$ and $\lambda^{j}$ are sensitive parameters related to attractiveness, activity duration and travel time on the space-time utility. Three place-time space-time accessibility measures are then developed to evaluate the accessibility of individuals living at location $i$. In the urban environment, a location $i$ may be covered by service areas of several facilities that provide the same types of activity. It should be noted that various sizes of service areas can be used for different facilities. These facilities constitute the facility choice set for an individual's activity participations, denoted by $F_{i}=\left\{f_{1}, \ldots, f_{n}\right\}$. The first place-based space-time accessibility measure, denoted by $C W A(i)$, adopts the consumer welfare aggregation (CWA) principle used in the conventional place-based accessibility measures. It is the summation of activity utilities provided by all facilities in the choice set $F_{i}$ :

$$
C W A(i)=\sum_{\forall f \in F_{i}} U_{f}(i)
$$


where $U_{f}(i)$ is the utility derived from activity participation at facility $f \in F_{i}$ defined in Eq. (6). This accessibility measure represents all possible activity utilities (welfares) enjoyed by an individual at location $i$.

In addition to the CWA principle, consumer welfare maximization (CWM) principle and random utility maximization (RUM) principle used in the space-time utility accessibility approach [14] are adopted. The second place-based space-time accessibility measure, denoted by $C W M(i)$, follows the CWM principle. Assuming the individual is a rational utility maximizer, he/she chooses only one facility $f \in F_{i}$ which provides the maximum activity utility for performing activities. Accordingly, the accessibility of individuals at location $i$ is measured as the maximum utility provided by a particular facility $f \in F_{i}$ :

$$
C W M(i)=\max _{\left\{f \in F_{i}\right\}}\left(U_{f}(i)\right)
$$

The third place-based space-time accessibility measure, denoted by RUM(i), adopts the RUM principle. Compared to the CWM principle, the RUM principle builds on the random utility theory, adding an unobservable random error component into the activity utility measure, i.e., Eq. (6). The error component of all facilities $\forall f \in F_{i}$ are assumed to follow independently and identically distributed Gumbel distributions. Accordingly, the mechanism of choosing a facility to maximize activity utilities can be formulated as a logit discrete choice model. Accessibility based on the RUM principle is therefore expressed as following logsum accessibility measure [15]:

$$
R U M(i)=\frac{1}{\lambda} \ln \left\{\sum_{\forall f \in F_{i}} \exp \left(U_{f}(i)\right)\right\}
$$

Unlike $C W M(i)$, this $R U M(i)$ measure is a summary indicator representing the expected maximum utility of the full facility choice set.

\section{Case Study}

This section presents a case study using real-world data collected in Wuhan which is one of the fast growing mega cities in China. This case study aims to demonstrate the applicability of the proposed accessibility evaluation framework. The case study is concerned with the accessibility to food service facilities in Wuhan. The data collected in this case study included a detailed digital road network, a large-scale taxi tracking data set for estimating travel time distributions, and information about dining facilities extracted from an online social media application. As shown in Figure 3, the Wuhan road network consisted of 19,306 nodes and 46,757 links. Real-world floating car data (FCD) was collected on a typical Thursday (September 3, 2009) to estimate traffic conditions of the Wuhan road network. The detailed method for estimating traffic conditions of Wuhan road network is documented in [21]. As shown, links shown in red represent congested links $(<20 \mathrm{~km} / \mathrm{h})$; yellow represents slightly congested links $(20-40 \mathrm{~km} / \mathrm{h})$; and green represents uncongested links $(>40 \mathrm{~km} / \mathrm{h})$. The figure shows that $9.7 \%$ of links in the Wuhan network were congested, mostly around seven commercial centres. 


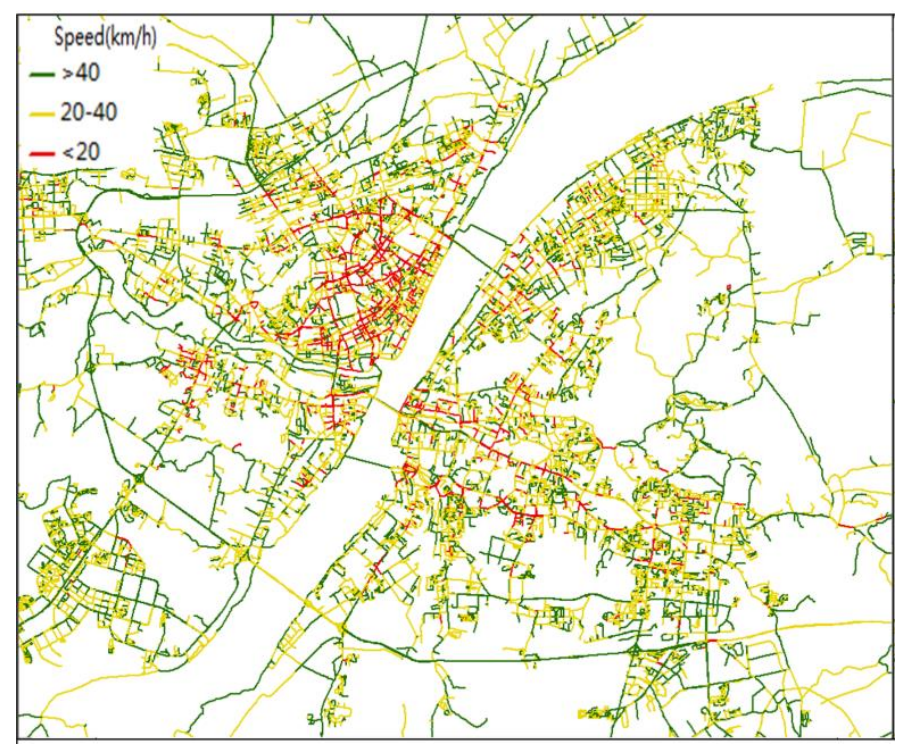

Fig. 3: Traffic condition of Wuhan network.



Fig. 4: Spatial distribution of food service facilities.

Data on food service facilities in Wuhan was collected from a location-based social networking website in China, named Jiepang (http://jiepang.com/). Jiepang, launched in 2010, is a popular web and mobile application, with five million users. In this study, 4,407 food service facilities were collected in September 2013. The spatial distribution of food service facilities is illustrated in Figure 4, which shows that dining facilities are not evenly distributed in the city but rather clustered at six commercial centres, especially in the Wuguang area.

Place-based space-time accessibility to food service facilities in Wuhan City was evaluated using the RUM principle, i.e., $R U M(i)$ defined in Eq. (10), for illustration. Figure 5 shows the resulting accessibility during the evening peak hour (6 p.m. -7 p.m.). As shown, the accessibility to dining facilities was not evenly distributed in Wuhan City. Compared to suburban areas, the values of RUM(i) were relatively high (over 300) in seven commercial centres. The Wuguang area had the highest value of $R U M(i)$ (over 800) due to its large number of food service facilities. Therefore, the proposed space-time accessibility measures can well represent the spatial heterogeneity of food service accessibility in Wuhan city. 


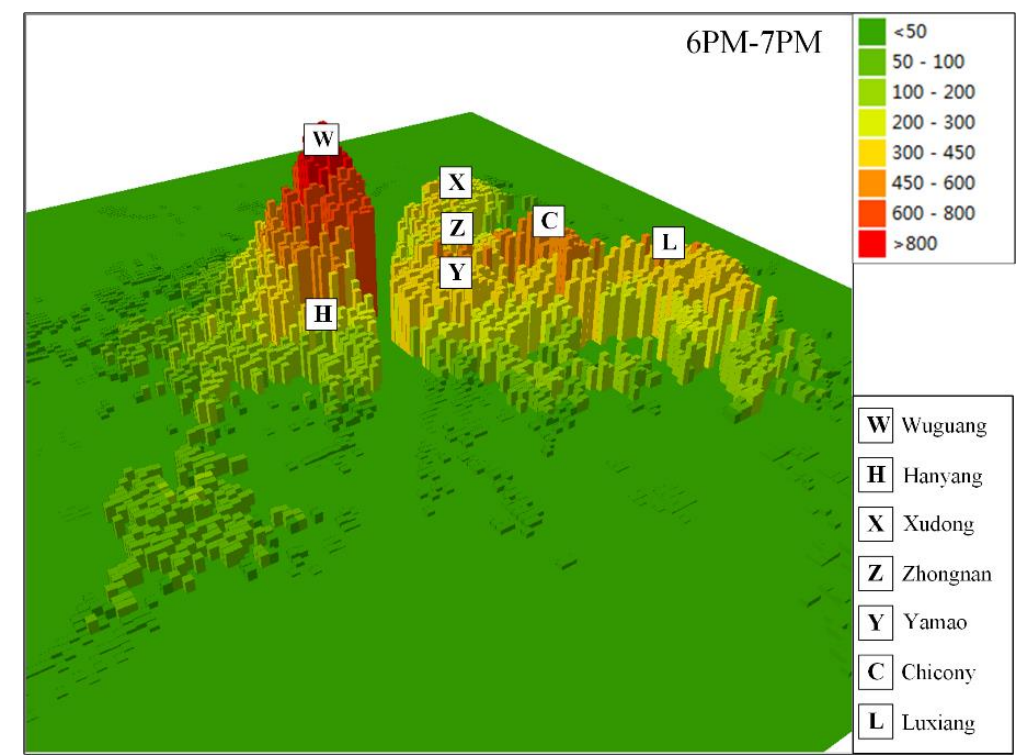

Fig. 5: Spatial distribution of accessibility to food service facilities in Wuhan city.

\section{Conclusion}

This study proposed new place-based accessibility measures with consideration of space-time effects for evaluating accessibility in large-scale study areas. The proposed framework reconciles conventional place-based accessibility measures with space-time utility approach. The accessibility of a location was measured with respect to activity duration, travel time and facility attractiveness. The individual choice behaviours amongst multiple alternatives are also explicitly considered by using three principles, including consumer welfare aggregation principle, consumer welfare maximization principle, and random utility maximization principle. To demonstrate the operationalization and applicability of the proposed place-based utility measures, a comprehensive case study is carried out in a mega city in China. The case study results indicated that the proposed space-time accessibility measures can incorporate time dimension into the place-based accessibility, in terms of activity duration and time-dependent traffic conditions.

Several directions for future research are worth noting. Firstly, the proposed accessibility measures are confined to private car mode only. The extension of the proposed accessibility evaluation framework to multi-modal networks is an interesting topic for fast-growing mega cities in China and Asia. Secondly, the accessibility to food services was examined mainly based on restaurants and other dining facilities. It is interesting to apply the proposed framework for evaluating accessibility to other types of urban opportunities, such as job and healthcare services. Last but not the least, the impacts of intelligent transport systems on the accessibility to urban services should also be further investigated.

\section{Acknowledgements}

The work described in this paper was jointly supported by the Research Grants Council of the Hong Kong Special Administrative Region, China (No. PolyU 152095/17E), the Research Institute for Sustainable Urban Development and Research Committee of the Hong Kong Polytechnic University (1-ZVEW and 4-ZZFY).

\section{References}

[1] M.-P. Kwan, and J. Weber, "Scale and accessibility: Implications for the analysis of land use-travel interaction," Applied Geography, vol. 28, no. 2, pp. 110-123, 2008.

[2] K. T. Geurs, and B. van Wee, "Accessibility evaluation of land-use and transport strategies: review and research directions," Journal of Transport Geography, vol. 12, no. 2, pp. 127-140, 2004.

[3] F. Liao, and B. van Wee, "Accessibility measures for robustness of the transport system," Transportation, vol. 44, no. 5, pp. 1213-1233, 2017.

[4] K. Lucas, B. van Wee, and K. Maat, "A method to evaluate equitable accessibility: combining ethical theories and accessibility-based approaches," Transportation, vol. 43, no. 3, pp. 473-490, 2016. 
[5] W. Yang, B. Y. Chen, X. Cao et al., "The spatial characteristics and influencing factors of modal accessibility gaps: A case study for Guangzhou, China," Journal of Transport Geography, vol. 60, pp. 21-32, 2017.

[6] D. W. S. Wong, and S. L. Shaw, "Measuring segregation: an activity space approach," Journal of Geographical Systems, vol. 13, no. 2, pp. 127-145, Jun. 2011.

[7] B. Y. Chen, Y. Wang, D. Wang et al., "Understanding the impacts of human mobility on accessibility using massive mobile phone tracking data," Annals of the American Association of Geographers, DOI:10.1080/24694452.2017.1411244., 2018.

[8] S. Silm, and R. Ahas, "Ethnic differences in activity spaces: A study of out-of-home nonemployment activities with mobile phone data," Annals of the Association of American Geographers, vol. 104, no. 3, pp. 542-559, May 4, 2014.

[9] M.-P. Kwan, and J. Weber, "Individual accessibility revisited: Implications for geographical analysis in the twentyfirst Century," Geographical Analysis, vol. 35, no. 4, pp. 341-353, 2003.

[10] M. P. Kwan, "Space-time and integral measures of individual accessibility: A comparative analysis using a pointbased framework," Geographical Analysis, vol. 30, no. 3, pp. 191-216, Jul, 1998.

[11] T. Neutens, T. Schwanen, F. Witlox et al., "Equity of urban service delivery: a comparison of different accessibility measures," Environment and Planning A, vol. 42, no. 7, pp. 1613-1635, Jul, 2010.

[12] H. J. Miller, "Place-based versus people-based geographic information science," Geography Compass, vol. 1, no. 3, pp. 503-535, 2007.

[13] T. Hägerstrand, "What about people in regional science?," Papers in Regional Science, vol. 24, no. 1, pp. 7-24, 1970 .

[14] H. J. Miller, "Measuring space-time accessibility benefits within transportation networks: Basic theory and computational procedures," Geographical Analysis, vol. 31, no. 2, pp. 187-212, Apr, 1999.

[15] B. van Wee, "Accessible accessibility research challenges," Journal of Transport Geography, vol. 51, pp. 9-16, 2016.

[16] A. Páez, R. G. Mercado, S. Farber et al., "Relative accessibility deprivation indicators for urban settings: Definitions and application to food deserts in Montreal," Urban Studies, vol. 47, no. 7, pp. 1415-1438, 2010.

[17] M. Delafontaine, T. Neutens, and N. V. d. Weghe, "A GIS toolkit for measuring and mapping space-time accessibility from a place-based perspective," International Journal of Geographical Information Science, vol. 26, no. 6, pp. 1131-1154, 2012.

[18] M. J. Widener, S. Farber, T. Neutens et al., "Using urban commuting data to calculate a spatiotemporal accessibility measure for food environment studies," Health \& Place, vol. 21, pp. 1-9, 2013.

[19] K. Fransen, T. Neutens, S. Farber et al., "Identifying public transport gaps using time-dependent accessibility levels," Journal of Transport Geography, vol. 48, pp. 176-187, Oct, 2015.

[20] M. J. Breheny, "The measurement of spatial opportunity in strategic planning," Regional Studies, vol. 12, no. 4, pp. 463-479, 1978.

[21] B. Y. Chen, C. Shi, J. Zhang et al., "Most reliable path-finding algorithm for maximizing on-time arrival probability," Transportmetrica B, vol. 5, no. 3, pp. 253-269, 2017. 